Ger J Exerc Sport Res 2020 · 50:308-319 https://doi.org/10.1007/s12662-020-00643-0 Eingegangen: 10. September 2019

Angenommen: 4. Januar 2020

Online publiziert: 24. Januar 2020

(c) Der/die Autor(en) 2020

\section{Tim Heemsoth ${ }^{1}$ D $\cdot$ Jonas Wibowo ${ }^{2}$}

${ }^{1}$ Fakultät für Erziehungswissenschaft, Arbeitsbereich Bewegung, Spiel und Sport, Universität Hamburg, Hamburg, Deutschland

${ }^{2}$ Fakultät für Human- und Sozialwissenschaften, Arbeitsbereich Sportpädagogik, Bergische Universität Wuppertal, Wuppertal, Deutschland

\title{
Fachdidaktisches Wissen von angehenden Sportlehrkräften messen
}

\section{Problemstellung}

Das fachdidaktische Wissen von Lehrkräften gilt im Allgemeinen und für das Fach Sport als wichtiger Bestandteil professioneller Kompetenz, die wiederum das unterrichtliche Handeln von Lehrkräften und das Erreichen von Unterrichtszielen bedeutsam bedingt (Baumert \& Kunter, 2006; Blömeke, Gustafsson, \& Shavelson, 2015; Heemsoth, 2016; Vogler, Messmer, \& Allemann, 2017; Wibowo \& Heemsoth, 2019). Befunde in mathematisch-naturwissenschaftlichen Fächern verweisen darauf, dass Lehrkräfte, die über ein hohes fachdidaktisches Wissen verfügen, ihre Schüler*innen in einem höheren Maße kognitiv aktivieren als Lehrkräfte mit geringerem fachdidaktischem Wissen und hierüber Leistungsentwicklungen der Lernenden erklärt werden können (z.B. Baumert et al., 2010). Vor diesem Hintergrund ist die zunehmende Bemühung in allen Fächern zu erklären, geeignete Instrumente zur Erfassung des fachdidaktischen Wissens zu entwickeln (z.B. Krauss et al., 2017). Für das Fach Sport befindet sich eine solche Entwicklung noch in den Anfängen (Heim \& Sohnsmeyer, 2015); insbesondere sind theoretische Konzeptionen bisher kaum empirisch hinsichtlich ihrer Konstruktvalidität geprüft worden. In der vorliegenden Untersuchung wird dieses Desiderat aufgegriffen. In Anlehnung an das Verständnis fachdidaktischen Wissens von Shulman (1986) wird das fachdidaktische Wissen von Sportlehrkräften vor allem mit den Wissensfacetten Lernende unterstützen, Schwierigkeiten erklären und Vorgehensweisen formulieren begründet, konzeptualisiert und in einen Test überführt. Neben einer Analyse der faktoriellen Struktur des Instrumentes wird untersucht, inwieweit das so erhobene Wissen durch die Abiturnote oder das Studiensemester vorhergesagt wird. Hierdurch werden als bedeutsam angenommene Erklärungen hinsichtlich der Entwicklung des fachdidaktischen Wissens überprüft (Kunter et al., 2013b). Folgende Forschungsfragen werden fokussiert: (A) Inwieweit lässt sich das fachdidaktische Wissen von Sportlehrkräften durch eine mehrfaktorielle Struktur modellieren? (B) Welche Zusammenhänge liegen zwischen einzelnen Faktoren vor? (C) Inwieweit wird das fachdidaktische Wissen durch die Abiturnote oder das Studiensemester vorhergesagt?

\section{Theoretischer Hintergrund}

\section{Fachdidaktisches Wissen als Teil professioneller Kompetenz}

Unter dem Professionswissen einer Lehrkraft kann das Wissen subsummiert werden, das notwendig ist, um erfolgreich zu unterrichten (Krauss, 2011). Dabei bilden in der kompetenzorientierten Professionsforschung neben dem Professionswissen weitere Dispositionen (motivationale Orientierungen, Überzeugungen und selbstregulative Fähigkeiten), situationsspezifische Fähigkeiten (Wahrnehmung, Interpretation, Ent- scheidungsfindung) und die Performanz die zentralen Bausteine professioneller Kompetenz einer Lehrkraft (Baumert \& Kunter, 2006; Blömeke et al., 2015). Hinsichtlich des Professionswissens wird gerade dem fachdidaktischen Wissen ein hoher Stellenwert beim erfolgreichen Unterrichten fachlicher Inhalte beigemessen, da gerade dieses es ermöglicht, anregende und an die Voraussetzungen der Lernenden angepasste Lernangebote anzubieten (Lange, Kleickmann, Tröbst, \& Möller, 2012). Es umfasst Wissen über das „Verständlichmachen von Fachinhalten“ (Krauss, 2011, S. 182) und wird teilweise als Integration von Fachwissen und allgemeinem pädagogischem Wissen angenommen. Eben diese drei Wissensformen (Fachwissen, fachdidaktisches Wissen, allgemeines pädagogisches Wissen) stellen mittlerweile eine über verschiedene Fächer weit verbreitete Topologie des Professionswissens von Lehrkräften dar, die auf Shulman (1986) zurückgeht. ${ }^{1}$

\footnotetext{
1 Shulman (1986) unterscheidet ursprünglich die Wissensbereiche Fachwissen, fachdidaktisches Wissen und curriculares Wissen. Später ergänzt er diese Wissensbereiche um das allgemeine pädagogische Wissen, das Wissen um die Psychologie des Lernens, das Organisationswissen und das erziehungsphilosophische und bildungstheoretische Wissen (Shulman, 1987). Von diesen Wissensbereichen haben sich in der gegenwärtigen Professionsforschung das Fachwissen, das fachdidaktische Wissen und das allgemeine pädagogische Wissen durchgesetzt (Baumert \& Kunter, 2006; Krauss, 2011).
} 
Zur Erfassung des fachdidaktischen Wissens ist in den letzten zehn Jahren fachübergreifend verstärkt eine Orientierung an proximalen Maßen zu beobachten, die vorzugsweise durch standardisierte Paper-and-Pencil-Tests erhoben werden. Dabei wird das fachdidaktische Wissen insbesondere durch „hypothetische Unterrichtsszenarios" (Krauss et al., 2017, S. 26) operationalisiert, auf die bezogen die Befragten nachfolgende Handlungen formulieren oder Handlungsalternativen bewerten sollen. Daran anknüpfende Befunde lassen sich vorsichtig wie folgt zusammenfassen: Während das Fachwissen eher geringe direkte oder nur indirekte Effekte auf die Leistungszuwächse von Schüler ${ }^{*}$ innen etwa im Fach Mathematik (Baumert et al., 2010), Biologie (Mahler, Großschedl, \& Harms, 2017) oder Sachunterricht (Ohle, Fischer, \& Kauertz, 2011) aufweist, lassen sich in diesen Studien die Leistungszuwächse durch das fachdidaktische Wissen bedeutsam vorhersagen. Die Vorhersage kann dabei teilweise durch Prozessmerkmale wie die kognitive Aktivierung und die individuelle Lernunterstützung erklärt werden (Baumert et al., 2010; Förtsch, Werner, von Kotzebue, \& Neuhaus, 2016). Im Rahmen eines Vergleiches mit weiteren als zentral angesehenen Merkmalen von Lehrkräften (konstruktivistische Überzeugungen, Unterrichtsenthusiasmus, selbstregulative Fähigkeiten) erweist sich das fachdidaktische Wissen zudem als stärkster Prädiktor für Leistungszuwächse (Kunter et al., 2013b). Zugleich finden sich auch Effekte in Bezug auf die affektiv-motivationale Entwicklung der Lernenden (Lange et al., 2012). Eine Erklärung für die relativ hohe prädiktive Kraft des fachdidaktischen Wissens könnte sein, dass es durch die Operationalisierung im Kontext von hypothetischen Unterrichtsszenarios eine starke Nähe zu den eigentlichen Unterrichtsprozessen aufweist (Baumgartner, 2018a).

Vor dem Hintergrund dieser Befunde ist zu erklären, dass derzeit verstärkt in allen Fächern Bemühungen zu beobachten sind, die darauf abzielen, das fachdidaktische Wissen durch entsprechende Testungen für das jeweilig Fach zugänglich zu machen. Dabei sind ma- thematisch-naturwissenschaftliche Fächer überproportional vertreten (z.B. Blömeke, Kaiser, \& Lehmann, 2010; Hill, Schilling, \& Ball, 2004; Kleickmann et al., 2014; Kunter et al., 2011; Mahler et al., 2017). Auch in einer relativ aktuell veröffentlichten fachübergreifenden Übersicht zu bisherigen Testentwicklungen entfallen 27 von 37 Nennungen auf mathematisch-naturwissenschaftliche Fächer (Krauss et al., 2017, S. 31/32), was wiederum Anlass für die Autoren ist, entsprechende Instrumente für ein breiteres Fächerspektrum zu entwickeln (Deutsch, Englisch, Latein, Physik, Musik und ev. Religion). Das Fach Sport taucht hier - wie auch in der Übersicht nicht auf. Dies verweist auf ein Desiderat, das auch von Heim und Sohnsmeyer (2015) formuliert wurde und dem sich die sportdidaktische Forschung erst in den letzten Jahren zugewandt hat. Dabei lassen hier insbesondere zwei Ansätze der deutschsprachigen Sportdidaktik identifizieren, die in Publikationsorganen mit wissenschaftlicher Qualitätssicherung (Peer-Review) veröffentlicht wurden. Diese werden im Folgenden detaillierter vorgestellt und eingeordnet. Darauf aufbauend werden Schlussfolgerungen für das eigene Vorgehen formuliert.

\section{Bisherige Konzeptualisierungs- ansätze für das Fach Sport}

Im Basler Ansatz (Vogler et al., 2017) wird das fachdidaktische Wissen von Sportlehrkräften erfasst, indem die Befragten im Rahmen eines Paper-andPencil-Tests zehn Unterrichtsfälle mit Problemcharakter lesen und jeweils aufgefordert werden, ein aus ihrer Sicht geeignetes unterrichtliches Handeln zu formulieren („Was antworten Sie? Wie fahren Sie mit dem Unterricht fort?" Vogler et al., 2017, S. 339). Es wird geprüft, inwieweit die offenen Antworten zuvor festgelegten Lösungsantworten entsprechen. Die Antworten werden dann zu Teilscores von insgesamt neun Faktoren summiert, die sich wiederum in Anlehnung an Scherler (2004) den drei Dimensionen „Inhalte präsentieren“, „Bedingungen organisieren“ und „Mit Schülerinnen Interagieren“ zuordnen lassen (Vogler et al., 2017, S. 338).
Das Instrument wird im Rahmen einer Studie mit insgesamt 113 Studierenden und 24 Lehrkräften (1. Erhebungszeitpunkt) sowie erneut 26 Studierenden (2. Erhebungszeitpunkt) eingesetzt, um Befunde zur Ausprägung des fachdidaktischen Wissens sowie zu Zusammenhängen zu spezifischen Variablen (va. Studiengang, Studienphase, Unterrichtserfahrung, Fachwissen) zu generieren. Insgesamt liefert der Basler Ansatz durch die Kontextualisierung mit umfangreichem Fallmaterial eine Modellierung im Sinne „hypothetische(r) Unterrichtsszenarios" (Krauss et al., 2017, S. 26). Der Einschätzung der Autoren, dass das fachdidaktische Wissen mit ihrem Instrument „reliabel und objektiv erhoben werden“ könne und es sich ,insgesamt als valide erwiesen“ habe (Vogler et al., 2017, S. 345/346), kann auf Basis der berichteten Befunde jedoch nur eingeschränkt gefolgt werden: So findet die Prüfung des Modells allein über eine Analyse von Kreuzkorrelationen der einzelnen Items statt, ohne dass der genaue Ablauf und Kennwerte berichtet werden. Eine übliche Prüfung der Konstruktvalidität durch exploratorische oder konfirmatorische Faktorenanalysen oder Analysen zur Reliabilität der einzelnen Faktoren wird nicht berichtet (Döring \& Bortz, 2016). Es fällt daher schwer, die weiteren Befunde zu Zusammenhängen einzelner Faktoren mit weiteren Variablen einzuordnen.

Im Kölner Ansatz (Meier, 2018) wird das fachdidaktische Wissen erfasst, indem die Befragten im Rahmen eines Paper-and-Pencil-Tests 81 sowohl geschlossene als auch offene Items beantworten. Die Items unterliegen einer Struktur auf zwei Ebenen: Auf inhaltlicher Ebene lassen sie sich in Anlehnung an Shulman (1986) dem Bereich Wissen über Repräsentationsformen und Instruktionsstrategien oder dem Bereich Wissen über inhaltsspezifische Lernschwierigkeiten und Schüler ${ }^{\star}$ innen-Kognitionen zuordnen. Im ersten Fall werden dabei insbesondere Aspekte eines Erziehenden Sportunterrichts aufgegriffen (Scheid \& Prohl, 2017), im zweiten Fall Lernschwierigkeiten in Bezug auf unterschiedliche Interessen, Aspekte der Körperlichkeit oder der Leistungsfähigkeit. Auf der Ebe- 
ne der kognitiven Anforderungen können die Items zudem in Anlehnung an Anderson und Krathwohl (2001) den Bereichen Erinnern, Verstehen/Analysieren oder Kreieren zugeordnet werden. Empirische Befunde wurden bis dato nicht publiziert. Aus theoretischer Perspektive kann festgestellt werden, dass der Ansatz eher keine kontextualisierte Erfassung des fachdidaktischen Wissens im Sinne hypothetischer Unterrichtsszenarios verfolgt und sich stattdessen eher auf deklaratives Faktenwissen konzentriert (s. etwa Meier, 2018, S. 80).

Insgesamt kann auf Basis der bisherigen Konzeptualisierungen und empirischen Befunde für das Fach Sport zusammengefasst werden: Wenn man das Wissen über die Vermittlung von Fachinhalten als zentralen Kern des fachdidaktischen Wissens auffasst (Lange et al., 2012; Shulman, 1986), so erscheint eine entsprechende Operationalisierung bis dato nicht hinreichend empirisch erprobt. Darüber hinaus werden bisher nurvereinzelt die Vorzüge rein kontextualisierter Operationalisierungen aufgegriffen (Vogler et al., 2017).

\section{Zur vorliegenden Untersuchung}

Der vorliegende Beitrag setzt an diesem Desiderat an und verfolgt das Ziel, ein entsprechendes Instrument zur Messung des fachdidaktischen Wissens von Sportlehrkräften zu entwickeln. Auf diesem Wege soll an die bisherige kompetenzorientierte Professionsforschung anderer Fächer angeknüpft werden, in denen das fachdidaktische Wissen als bedeutsamer Prädiktor für unterrichtliches Handeln und das Erreichen von Unterrichtszielen angenommen und empirisch bestätigt wurde (z. B. Baumert et al., 2010; Lange et al., 2012; Mahler et al., 2017).

\section{Gestaltungsmerkmale für die Konzeption des fachdidaktischen Wissens}

Bei der Konzeption des fachdidaktischen Wissens werden in diesem Beitrag drei Gestaltungsmerkmale berücksichtigt. Die Items umfassen erstens realistische Unterrichtsszenarios, in denen zweitens bewegungsbezogene Aufgaben in Bewegungsfeldern thematisiert werden.

Ger J Exerc Sport Res 2020 · 50:308-319 https://doi.org/10.1007/s12662-020-00643-0

(c) Der/die Autor(en) 2020

\section{T. Heemsoth · J. Wibowo}

\section{Fachdidaktisches Wissen von angehenden Sportlehrkräften messen}

\section{Zusammenfassung}

Das fachdidaktische Wissen gilt in der lehrkraftbezogenen Professionsforschung als zentrale Komponente professioneller Kompetenz, durch die die Qualität der Unterrichtshandlungen und das Erreichen von Unterrichtszielen vorhergesagt werden kann. Für das Fach Sport liegen zwar vereinzelt empirische Ansätze zur Erfassung des fachdidaktischen Wissens vor, gleichwohl fehlt es bis dato an einem Instrument, dessen faktorielle Struktur empirisch geprüft wurde. Im vorliegenden Beitrag wird das fachdidaktische Wissen über 40 Items erhoben, in denen im Rahmen realistischer Unterrichtsszenarios bezogen auf acht Bewegungsfelder dazu aufgefordert wird, Lernende zu unterstützen, Schwierigkeiten zu erklären oder Vorgehensweisen zu formulieren. Nach einer zufriedenstellenden Expert*innen-Befragung wurden die
Items von 290 Sportlehramtsstudierenden beantwortet. Die Befunde deuten darauf hin, dass sich das fachdidaktische Wissen zufriedenstellend über die drei Faktoren Lernende unterstützen, Schwierigkeiten erklären sowie Vorgehensweisen formulieren abbilden lässt. Sie korrelieren im mittleren bis hohen Bereich. Darüber hinaus wird das fachdidaktische Wissen bedeutsam durch die Anzahl der Studiensemester, nicht aber durch die Abiturnote vorhergesagt, was die Qualifikationshypothese bekräftigt. Insgesamt liegt eines der wenigen empirisch überprüfen Instrumente für das fachdidaktische Wissen angehender Sportlehrkräfte vor.

Schlüsselwörter Professionsforschung · Professionelle Kompetenzen - Sportunterricht - Bewegungsfelder . Konfirmatorische Faktorenanalyse

\section{Assessment of physical education teachers' pedagogical content knowledge}

\section{Abstract}

Research on teacher education assumes pedagogical content knowledge (PCK) to be a core component of professional competence that predicts both the quality of teaching and the achievement of teaching goals. In the field of physical education, there are some empirical approaches to describe PCK; however, an instrument assessing PCK, which factor structure has been empirically analyzed, is still missing. The current study assesses PCK via 40 items. All items comprise realistic teaching scenarios within one of eight movement fields and demand to support learners, to explain learning difficulties or to formulate appropriate instructions. After a satisfactory expert rating, 290 students studying to be physical education teachers answered the items. Results indicate that PCK can be measured by the factors supporting learners, explaining learning difficulties and formulating learning instructions. The factors correlated with medium to high effect sizes. Furthermore, the teacher students' semester predicted PCK, whereas the grade point average did not. This finding supports the qualification hypothesis. Overall, with regard to students learning to be physical education teachers, the study provides one of the few instruments that has been empirically tested.

\section{Keywords}

Research on professional development . Professional competencies - Physical education - Movement fields - Confirmatory factor analysis
Drittens bezieht sich ein jedes Item, in Anlehnung an domänenübergreifende Konzeptionen (Shulman, 1986), auf eine von drei Wissensfacetten, die unterschiedliche Aspekte des Verständlichmachens von Fachinhalten aufgreifen. Während das erste Gestaltungsmerkmal zuvor bereits diskutiert wurde, wird auf die letzten beiden im Folgenden detaillierter eingegangen, wobei auch fachdidaktische Bezüge herausgearbeitet werden: So kann das zentrale Ziel von Sportunterricht aus der sportdidaktischen Perspektive eines erziehenden Sportunterrichts als „Bewegungsbildung im Horizont allgemeiner Bildung“ 
(Prohl, 2017a, S. 70) beschrieben werden; dies gelingt dann, wenn über die sachliche Auseinandersetzung mit bewegungsbezogenen Inhalten (Erziehung zum Sport) fachspezifische Bildungsprozesse angestoßen werden, die zugleich zur Persönlichkeitsentwicklung beitragen (Erziehung durch Sport). Dafür solle sowohl auf der Inhalts- wie auch der Vermittlungsebene eine fachlich-didaktisch strukturierte Öffnung stattfinden. Auf der Inhaltsebene lässt sich in diesem Kontext eine zunehmende Orientierung an bewegungsbezogenen Aufgaben in Bewegungsfeldern feststellen, die die kulturellen Erscheinungsformen von Bewegung, Spiel und Sport nach strukturellen Gesichtspunkten bündeln (s. auch Laging, 2009). Eine solche Orientierung nimmt heute auch eine starke Position im Rahmen deutscher Sportlehrpläne ein (Prohl \& Krick, 2006), was auch auf die (normative) Bedeutung eines auf Bewegungsfelder bezogenen fachdidaktischen Wissens von Sportlehrkräften verweist. Insgesamt wird der Bedeutung von Bewegungsfeldern durch das zweite Gestaltungsmerkmal Rechnung getragen. Auf der Vermittlungsebene bedeutet die Öffnung eine Abkehr einer direktiven technikzentrierten Vorgehensweise hin zu einem Sportunterricht, der stärker auf das selbstständige Lösen von Bewegungsproblemen abzielt (Prohl, 2017b). Damit einher geht eine gesteigerte Notwendigkeit, die Lernenden während ihrer Auseinandersetzung mit Bewegungsproblemen lehrkraftseitig zu begleiten und $\mathrm{zu}$ unterstützen. Dafür kann angenommen werden, dass Sportlehrkräfte Wissen über geeignete Erklärungs- und Unterstützungsmöglichkeiten in Bezug auf den Lerninhalt sowie Wissen über Lernschwierigkeiten haben sollten. Hierauf bezogene Wissensfacetten finden sich in Anlehnung an Shulman (1986) domänenübergreifend bei Konzeptualisierungen zum fachdidaktischen Wissen (Baumert \& Kunter, 2006; Blömeke et al., 2015; Krauss, 2011; für den Sportunterricht s. auch Meier, 2018). Darüber hinaus findet sich vielfach eine dritte Wissensfacette, die über die Eckpunkte des didaktischen Dreiecks begründet werden kann (Krauss et al., 2017). So wird auch für den Sportunter- richt angenommen, dass neben einem Wissen über Erklärungs- und Unterstützungsmöglichkeiten (Eckpunkt Inhalt) und dem Wissen über Schwierigkeiten mit Fachinhalten (Eckpunkt Lernende) auch ein Wissen über umfangreichere unterrichtliche Herangehens- bzw. Vorgehensweisen mit unterschiedlich starker Lenkung der Lehrkraft (Eckpunkt Lehrkraft) notwendig ist (Heemsoth, 2016). Dabei ist zu betonen, dass unterschiedliche didaktische Begründungen eine Vorgehensweise mit eher starker oder eher geringer Lenkung nahelegen, es jedoch für eine Sportlehrkraft notwendig erscheint, möglichst unterschiedliche Vorgehensweisen zu kennen, um entsprechend der Lerngruppe und den Gegebenheiten angemessen zu handeln (Größing, 2007). Insgesamt werden durch das dritte Gestaltungsmerkmal daher die drei Wissensfacetten Lernende unterstützen, Schwierigkeiten erklären und Vorgehensweisen formulieren unterschieden.

\section{Entwicklung professioneller Kompetenz}

Neben einer Prüfung der faktoriellen Validität des Instrumentes wird im Rahmen der Entwicklung neuer Instrumente empfohlen, auch theoretisch angenommene Zusammenhänge $\mathrm{zu}$ weiteren bedeutsamen Variablen zu untersuchen (zur Kriteriumsvalidität s. etwa Döring \& Bortz, 2016). Dafür werden in dieser Studie die Abiturnote und die Semesterzahl fokussiert, mit denen unterschiedliche Erklärungen zur Entwicklung professioneller Kompetenz verbunden sind. Es lassen sich zwei zentrale Hypothesen unterscheiden (Kunter, Kleickmann, Klusmann, \& Richter, 2013a): Auf der einen Seite wird im Rahmen der Hypothese der persönlichen Eignung angenommen, dass stabile kognitive Fähigkeiten dafür verantwortlich sind, inwieweit eine (angehende) Lehrkraft erfolgreich unterrichtet (s. dazu auch Kennedy, Ahn, \& Choi, 2008). In diesem Verständnis sollte sich auch die professionelle Kompetenz (und das fachdidaktische Wissen) vor allem durch die persönliche Eignung erklären lassen. Auf der anderen Seite wird im Rahmen der Qualifikationshypothese angenommen, dass erst spezifische Ausbildungsangebote, die darauf abzielen, professionsbezogene Konzepte und Prinzipien (und somit auch fachdidaktisches Wissen) zu vermitteln, dazu führen, dass eine Lehrkraft erfolgreich unterrichtet (Bromme, 2001; Shulman, 1987). Bezüglich der ersten Hypothese ist die Befundlage durchwachsen: Während teilweise kleine positive Zusammenhänge zwischen generellen kognitiven Fähigkeiten der Lehrkraft (vielfach gemessen in Form der Abiturnote) und Schüler*innen-Leistungen festgestellt werden konnten (Aloe \& Becker, 2009), werden an anderer Stelle keine Zusammenhänge berichtet (Grönqvist \& Vlachos, 2008; Yeh, 2009). Hingegen scheint die Befundlage im Sinne der Qualifikationshypothese eindeutiger: Es zeigt sich, dass sich sowohl das Fachwissen als auch das fachdidaktische Wissen größtenteils während des Studiums und des Referendariats entwickelt, während im Verlauf der Berufsausübung kaum weitere Zuwächse festzustellen sind (Kleickmann et al., 2013). Auch erste Studien im Kontext der Sportlehrkräftebildung zeigen, dass durch spezifische Ausbildungsformate handlungsnahe Kompetenzen wie das unterrichtliche Planen (Heemsoth \& Kleickmann, 2018) oder gar unterrichtliche Handlungen (Baumgartner, 2018b) verbessert werden können. In diesem Sinne wird das hier entwickelte Instrument eingesetzt, um entsprechende Zusammenhänge zwischen dem fachdidaktischen Wissen, generellen kognitiven Fähigkeiten (in Form der Abiturnote) und der Ausbildungsdauer (in Form der Semesterzahl) zu untersuchen.

\section{Forschungsfragen und Hypothesen}

Vor dem Hintergrund der zuvor beschriebenen Konzeption sowie den Überlegungen zur Entwicklung des fachdidaktischen Wissens ergeben sich folgende Forschungsfragen und Hypothesen:

(A) Inwieweit lässt sich das fachdidaktische Wissen von Sportlehrkräften durch eine mehrfaktorielle Struktur modellieren? Es wird angenommen, dass sich das fachdidaktische Wissen durch die Faktoren Lernende unterstützen, Schwierigkei- 
In Ihrer 7. Klasse soll der Tic-Tac erlernt werden, eine Basistechnik im Parkoursport, um z. B. über einen Kasten zu springen (vgl. Abb.). Einern Schüler gelingt es nicht, sich ausreichend von der Wand abzudrücken. Welche der folgenden Vorübungen ist geeignet, um den Lernprozess zu unterstützen?
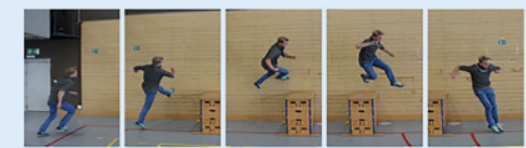

Handstütz auf dem Kasten, ein Schritt gegen die

Wand und Aufknien aufd em Kasten.

Nutzung eines Minitrampolins vor dem Hindernis

als SQrunghilfe.

Springen aus einer Absprungzone nahe am

Hindernis.

Handstütz auf dem Kasten, ein oder mehrere

Schritte an der Wand und Überspringen des

Kastens ohne Fußkontakt auf dem Kasten.

Beidbeiniges Abspringen an die Wand.

Ihre 9. Klasse soll das schnelle Überlaufen von Hindernissen erlernen und zentrale Bewegungsmerkmale benennen können. Möglich sind folgende Vorgehensweisen:

a) Übt die Bewegung so, wie ich sie euch vormache. Achtet dabei auf die genannten Merkmale.

b) Erprobt, wie ihr am schnellsten über die Hindernisse kommt und erklärt, worauf man achten muss.

Formulieren Sie eine Vorgehensweise mit einer (mehreren) Aufgabenstellung(en), die den Schülerinnen und Schülern mehr Freiraum als in a) lässt, aber stärker inhaltlich strukturiert als in b ). Beschreiben Sie konkret, welche Strategien, Merkmale, Übungen etc. Sie bei ihrer Vorgehensweise berücksichtigen.
Sie möchten in einer 8. Klasse den Inhalt

Judo einführen. Was sind typische

Probleme für Schülerinnen und Schüler? Ja Nein

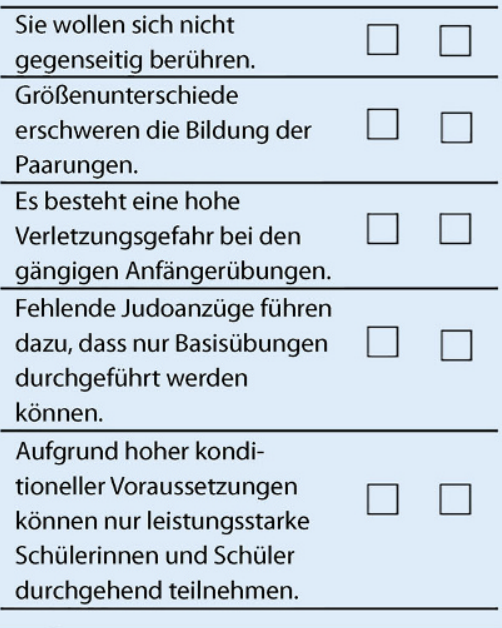

Paarungen.
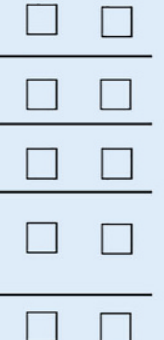

Ja Nein 
Tab. 1 Beispiel eines Kodiermanuals für das Item „Hindernisse“ der Wissensfacette Vorgehensweisen formulieren

\begin{tabular}{|c|c|c|}
\hline Kriterium & Allgemeine Beschreibung & Beispielantwort (Auswahl) \\
\hline $\begin{array}{l}\text { 1a: Inhaltliche } \\
\text { Strukturierung }\end{array}$ & $\begin{array}{l}\text { Es werden inhaltliche Entscheidun- } \\
\text { gen durch die Lehrkraft vorgenom- } \\
\text { men }\end{array}$ & $\begin{array}{l}\text { "Die Schüler*innen konzentrieren sich } \\
\text { auf die Höhe der Flugkurve über den } \\
\text { Hindernissen." }\end{array}$ \\
\hline $\begin{array}{l}1 \mathrm{~b} \text { : Methodi- } \\
\text { sche Struktu- } \\
\text { rierung }\end{array}$ & $\begin{array}{l}\text { Die Lehrkraft setzt Entscheidungen } \\
\text { bezüglich Zielen, Lernwegen oder } \\
\text { Lernpartner fest }\end{array}$ & $\begin{array}{l}\text { „Auf einem Arbeitsblatt werden die } \\
\text { verschiedenen Aufträge formuliert.” }\end{array}$ \\
\hline $\begin{array}{l}\text { 2a: Inhaltliche } \\
\text { Entscheidun- } \\
\text { gen }\end{array}$ & $\begin{array}{l}\text { Den Lernenden werden Entscheidun- } \\
\text { gen einer inhaltlichen Fokussierung } \\
\text { gewährt }\end{array}$ & $\begin{array}{l}\text { „Probiert verschiedene Lösungen } \\
\text { aus, die für ein schnelles Überqueren } \\
\text { sinnvoll sind." }\end{array}$ \\
\hline $\begin{array}{l}\text { 2b: Methodi- } \\
\text { sche Entschei- } \\
\text { dungen }\end{array}$ & $\begin{array}{l}\text { Den Lernenden werden Entschei- } \\
\text { dungen bezüglich Lernwege oder } \\
\text { Sozialform usw. gewährt }\end{array}$ & $\begin{array}{l}\text { „Die Lernenden suchen sich einen } \\
\text { Partner." }\end{array}$ \\
\hline
\end{tabular}

einzelne Probleme auftreten können oder inwieweit Lernschwierigkeiten auf bestimmte Erklärungen zurückgeführt werden können (• Abb. 1, rechts oben). Die Entscheidung über die Richtigkeit der Antworten in den ersten beiden Wissensfacetten erfolgte ebenfalls auf Basis bewegungsfeldbezogener Literatur (und wurde durch eine Expert ${ }^{*}$ innenBefragung überprüft, s. nachfolgender Abschnitt). Schließlich werden bei Items der Wissensfacette Vorgehensweisen formulieren sowohl ein unterrichtliches Ziel formuliert als auch zwei unterschiedliche Vorgehensweisen skizziert, die sich im Grad ihrer Lenkung durch die Lehrkraft unterscheiden. In der ersten Vorgehensweise wird ein durch die Lehrkraft stark strukturiertes und gelenktes Vorgehen und in der zweiten Vorgehensweise ein offenes, kaum strukturiertes Vorgehen mit großer Selbstbestimmung der Lernenden skizziert. Im Anschluss soll eine Vorgehensweise mittlerer Lenkung formuliert werden, die sich im Grad der Lenkung zwischen den beiden skizzierten Vorgehensweisen bewegt (• Abb. 1, unten). So wird auf der einen Seite im Sinne Prohls (2017a) ein Wissen über eine geöffnete Vermittlung berücksichtigt, die jedoch auch lehrkraftseitige Strukturierungshilfen umfasst; denn fachübergreifende Befunde weisen darauf hin, dass eine maximale Selbstbestimmung zumindest einen Teil der Lernenden überfordern kann (Kirschner, Sweller \& Clark, 2006).

\section{Expert*innen-Befragung}

Nach den konzeptuellen Vorarbeiten wird zur Überprüfung der Inhaltsvalidität empfohlen, das entwickelte Instrument einer Beurteilung durch Fachexpert*innen auszusetzen (Döring \& Bortz, 2016, S. 446). In der vorliegenden Studie wurden dafür Urteile von insgesamt $N=28$ Expertinnen (47\%) und Experten (53\%) eingeholt (s. auch Wibowo \& Heemsoth, 2019). Von den 28 Personen vertreten 15 Personen die Sportdidaktik in Lehre und Forschung an einer deutschsprachigen Universität, 13 Personen sind als Fachseminarleiterinnen bzw. Fachseminarleiter in der Ausbildung im Referendariat im Fach Sport tätig. Sie waren im Durchschnitt $M=48,60$ Jahre alt $(S D=9,32)$ und hatten eine durchschnittliche Berufserfahrung von $M=13,41$ Jahren $(S D=8,13)$. Als weitere Auswahlkriterien galten eine Berufserfahrung von mindestens 5 Jahren und ein abgeschlossenes Lehramtsstudium im Fach Sport. Im Rahmen einer Onlinebefragung mit der Software Unipark (Questback GmbH, 2018) beantworteten sie zunächst jedes Item selbst und wurden direkt im Anschluss an jedes Item aufgefordert, die Relevanz („Der hier beschriebene Kontext ist für den Sportunterricht relevant“) sowie die Plausibilität (geschlossene Items: „Die Zuordnung richtiger und falscher Antworten scheint mir plausibel“; offene Items: „Es gelingt mir, eine geeignete Antwort zu formulieren“) des Items auf einer 5-stufigen Likert-Skala einzuschätzen $(0=$ trifft nicht $z u, 1=$ trifft eher nicht $z u, 2=$ teils-teils, $3=$ trifft eher $z u, 4=$ trifft $z u)$. Abschließend wurden in einer offenen Frage Verbesserungsvorschläge erbeten („Ich habe folgende Hinweise/ Anregungen").

\section{Bewertung der Antworten der Studierenden}

Bei den geschlossenen Items der ersten beiden Wissensfacetten wurde für jedes richtig gesetzte Kreuz ein Punkt vergeben, sodass maximal fünf Punkte pro Item zu erzielen waren. Die erzielten Punkte pro Item wurden jeweils durch 5 dividiert, sodass der Mittelwert eines Items einen Wert zwischen 0 und 1 einnimmt und somit seiner Schwierigkeit entspricht.

Die Grundlage für die Bewertung der offenen Studierendenantworten im Bereich Vorgehensweisen formulieren ist eine inhaltlich strukturierende qualitative Inhaltsanalyse (Kuckartz, 2016) der offenen Antworten der Expert*innen. Die thematischen Hauptkategorien wurden theoriebasiert (s. Abschn. ,Testkonstruktion") aus dem Anspruch entwickelt, mit dieser Wissensfacette das Vermögen der Proband*innen zu erfassen, eine Vorgehensweise mittlerer Lenkung in einem konkreten Inhaltsbereich zu planen. Als notwendige Voraussetzung dafür wird angenommen, dass eine Lehrkraft fachinhaltliche (1) Strukturierungshilfen bereitstellen kann und (2) durch das Ermöglichen von Entscheidungen eine Öffnung und fachinhaltliche Aktivierung ermöglicht. Diese Voraussetzungen stellen erste zentrale Kategorien dar. Nach der ersten Kodierung eines Teilmaterials (ca. 10\% der Antworten, s. dazu auch Kuckartz, 2016, S. 80) wurden die beiden Kategorien hinsichtlich (a) Inhalte und (b) $\mathrm{Me}$ thoden ausdifferenziert. Hierdurch entstehen vier thematische Hauptkategorien (1a, 1b, 2a, 2b), die durch die Auswertung der Antworten der Expert*innen für jede Aufgabe inhaltlich ausgefüllt wurden. Dafür wurden alle Antworten durch die zwei späteren Raterinnen kodiert und zusammengefasst.

Neben einer allgemeinen Beschreibung (• Tab. 1) lassen sich die Kategorien exemplarisch an dem Item Hindernisse illustrieren (• Abb. 1, unten): Für eine 
Tab. 2 Itemkennwerte der entwickelten Skalen

\begin{tabular}{|c|c|c|c|c|c|c|}
\hline \multirow[t]{3}{*}{ Wissensfacette (Itemzahl) } & \multicolumn{2}{|c|}{ Expert*innen-Befragung } & \multicolumn{4}{|c|}{ Studierendenbefragung } \\
\hline & \multirow{2}{*}{$\begin{array}{l}\text { Relevanz } \\
M(S D)\end{array}$} & \multirow{2}{*}{$\begin{array}{l}\text { Plausibilität } \\
M(S D)\end{array}$} & \multicolumn{2}{|c|}{ Deskriptive Ergebnisse } & \multirow{2}{*}{$\begin{array}{l}\text { Interne } \\
\text { Konsistenz } \\
\text { a }\end{array}$} & \multirow{2}{*}{$\begin{array}{l}\text { Interraterreliabilität } \\
\text { к: } M(S D)\end{array}$} \\
\hline & & & $M(S D)$ & Range & & \\
\hline Lernende unterstützen (16) & $3,35(0,94)$ & $2,89(1,15)$ & $0,69(0,08)$ & $0,53-0,82$ & 0,60 & - \\
\hline Schwierigkeiten erklären (16) & $3,26(1,02)$ & $3,01(1,07)$ & $0,76(0,08)$ & $0,64-0,90$ & 0,66 & - \\
\hline Vorgehensweisen formulieren (8) & $3,54(0,74)$ & $3,24(0,87)$ & $0,59(0,14)$ & $0,52-0,72$ & 0,78 & $\begin{array}{l}0,80(0,04) \\
\text { Range } 0,74-0,85\end{array}$ \\
\hline \multicolumn{7}{|c|}{$\begin{array}{l}\text { Bem.: Skalierung der Augenscheinvalidität (Relevanz für den Sportunterricht) und Plausibilität: } 0=\text { trifft nicht } z u ; 1=\text { trifft eher nicht } z u, 2=\text { teils-teils, } \\
3=\text { trifft eher } z u, 4=\text { trifft zu } \\
\text { Möglicher Range der Studierendenantworten: } 0,00-1,00\end{array}$} \\
\hline
\end{tabular}

inhaltliche Strukturierung (1a) werden von den Expert ${ }^{\star}$ innen hier etwa konkrete Problemstellungen mit inhaltlichem Fokus vorgeschlagen („Wie schaffe ich einen gleichbleibenden Rhythmus?"), oder es wird das Einbringen von Bewegungsmerkmalen oder akustischen und optischen Hilfen empfohlen. Für eine methodische Strukturierung (1b) wird der Einsatz von Arbeitsblättern oder Gruppenarbeit vorgeschlagen, oder es wird der Durchlauf einer (konkret benannten) Übungsreihe empfohlen. Um inhaltliche Entscheidungen (2a) $\mathrm{zu}$ ermöglichen werden etwa Erprobungsaufträge vorgeschlagen („Probiert verschiedene Lösungen aus, die für ein schnelles Überqueren sinnvoll sind"). Methodische Entscheidungen (2b) liegen etwa dann vor, wenn Ziele, Wege, Übungen, Trainingsschwerpunkte, Zeiten oder Sozialformen zu einer Aufgabenstellung durch die Schüler*innen selbst bestimmt werden können. Für jedes Item wurde ein eigenes Kodiermanual erstellt.

Da aus der fachdidaktischen Perspektive eines erziehenden Sportunterrichts sowohl solche Elemente als sinnvoll erachtet werden, die den Unterricht strukturieren, als auch solche, die einen Spielraum für Entscheidungen ermöglichen, werden Antworten höher numerisch bewertet, wenn sie mehr Kategorien abdecken (pro Kategorie 1a, 1b, 2a und 2b jeweils 0,25 Punkte). Da hier insgesamt hoch inferente Items vorliegen, wurden $33 \%$ der Antworten durch zwei unabhängige Rater doppelt kodiert. Die InterraterÜbereinstimmung war zufriedenstellend (Cohens Kappa im Bereich 0,74-0,85; $M=0,80 ; S D=0,04 ; \bullet$ Tab. 2).

\section{Studierendenstichprobe und Untersuchungsdurchführung}

An der Haupterhebung nahmen insgesamt 290 Studierende des Faches Sport teil, die im Rahmen des Bachelor- (57\%) oder Masterstudiums (41\%) eingeschrieben waren. $68 \%$ der Studierenden waren mindestens im 5. Bachelorsemester. Die Studentinnen (58\%) und Studenten (42\%) waren durchschnittlich $M=24,9$ Jahre alt $(S D=4,0)$ und haben im Durchschnitt $M=5,9$ Semester $(S D=2,1)$ studiert. Ihre durchschnittliche Abiturnote beträgt $M=2,4(S D=0,5)$. Die Onlinebefragung der Studierenden mittels Unipark erfolgte im Rahmen einer Hausaufgabe in Lehrveranstaltungen in der sportpädagogischen oder sportdidaktischen Lehre und dauerte durchschnittlich 82 min. Die Studierenden wurden vorab dazu aufgefordert, die Fragen nur auf Basis ihres vorhandenen Wissens zu beantworten.

\section{Auswertungsverfahren}

Auf Basis der Studierendenantworten wurden Strukturgleichungsmodelle mit MPlus 8 (Muthén \& Muthén, 2012) durchgeführt. Als Schätzmethode wurde der für den gewählten Analysetypen („general“) gängige Maximum Likelihood-Schätzer (ML) verwendet. Durch Berücksichtigung des Full-InformationMaximum-Likelihood-Schätzverfahrens (FIML) konnten fehlende Werte modellbasiert geschätzt werden.

Neben dem $\chi^{2}$-Test werden robustere Maße zur Bewertung der Modellgüte hinzugezogen: Demnach stellt ein RSMEA unter 0,08 einen akzeptablen und unter 0,05 einen exzellenten Fit dar. Für den SRMR werden Grenzwerte bis 0,10 als akzeptabel bzw. unter 0,05 als exzellent angesehen. Die inkrementellen Fitindizes CFI und TLI stellen über 0,90 einen akzeptablen und über 0,95 einen exzellenten Fit dar (Schermelleh-Engel, Moosbrugger, \& Müller, 2003). Insgesamt sollten einzelne Pfade ein Signifikanzniveau von $p<0,05$ aufweisen. Dabei gehen die Items nicht grundsätzlich als einzelne manifeste Indikatoren, sondern zum Teil als Parcel in die Analyse ein: Jeweils die beiden Items der Wissensfacetten Lernende unterstützen und Schwierigkeiten erklären zum gleichen Bewegungsfeld werden $\mathrm{zu}$ einem Indikator durch Mittelwertbildung zusammengefasst. Dies ist nach Bandalos (2002) dann adäquat, wenn die Fragestellung auf die Beziehung von Konstrukten fokussiert, was in dieser Untersuchung der Fall ist. Das Parcelling hat den Vorteil, dass die zu schätzenden Parameter auf diese Weise reduziert werden, aufgrund derer es bei komplexeren Modellen oft zur Modellablehnung kommt (in diesem Fall lägen 40 Items und entsprechend viele Freiheitsgerade vor). Dieser Vorteil lässt sich schließlich auch darauf zurückführen, dass den geparcelten Skalen eher eine multivariate Normalverteilung zu Grunde liegt (Bandalos, 2002; Little, Cunningham, Shahar, \& Widaman, 2002).

Um zu prüfen, ob sich das fachdidaktische Wissen durch eine mehrfaktorielle Struktur abbilden lässt (Hypothese 1), wurden konfirmatorische Faktorenanalysen durchgeführt. Es wurde ein 3-Faktor-Modell, bei dem die drei Wissensfacetten getrennt voneinander modelliert wurden, mit einem G-Faktor- 


\section{Tab. 3 Modellfit-Indizes der betrachteten Modelle}

\begin{tabular}{|c|c|c|c|c|c|c|c|c|c|}
\hline Modell & $x^{2}$ & Df & $p$ & CFI & TLI & RMSEA & SRMR & AIC & BIC \\
\hline G-Faktor-Modell & 459,72 & 252 & $<0,01$ & 0,75 & 0,72 & 0,05 & 0,07 & $-5160,55$ & $-4896,32$ \\
\hline 3-Faktor-Modell & 319,71 & 249 & $<0,01$ & 0,91 & 0,91 & 0,03 & 0,06 & $-5295,10$ & $-5019,86$ \\
\hline 2-Faktor-Modell & 330,07 & 251 & $<0,01$ & 0,90 & 0,90 & 0,03 & 0,06 & $-5288,20$ & $-5020,30$ \\
\hline
\end{tabular}

Modell, bei dem alle Items auf einem einzigen Faktor laden, verglichen. Die Differenz der Modelle wurde anhand der $\chi^{2}$ Differenz überprüft, außerdem werden deskriptive Modellparameter miteinander verglichen (AIC und BIC). Für die Analyse der Zusammenhänge zwischen einzelnen Faktoren (Hypothese 2) sowie der Vorhersage einzelner Faktoren des fachdidaktischen Wissens durch die manifesten Variablen Abiturnote sowie Semesterzahl (Hypothese 3) werden latente Korrelationen berichtet. Dabei gilt ein Wert von $0,10<r<0,30$ als kleiner Effekt, $0,30<r<0,50$ repräsentiert einen mittleren und $r>0,50$ einen großen Effekt (Cohen, 1988).

\section{Ergebnisse}

\section{Vorabanalysen: deskriptive Werte und psychometrische Gütekriterien}

Zunächst kann anhand der Expert*innen-Befragung zur Relevanz festgestellt werden, dass den Items (bei einem theoretischen Mittelwert von 2) insgesamt eine eher hohe Relevanz für den Sportunterricht beigemessen wird. Alle Wissensfacetten erreichen im Durchschnitt Werte über 3 (•Tab. 2). Auch die Plausibilität der Antworten kann insgesamt als hoch eingeschätzt werden. Während die Items der Wissensfacette Lernende unterstützen durchschnittlich knapp unter $3(M=2,89 ; S D=1,15)$ eingeschätzt werden, werden beide weiteren Wissensfacetten durchschnittlich über 3 eingeschätzt (Schwierigkeiten erklären: $M=3,01 ; \quad S D=1,07 ; \quad$ Vorgehensweisen formulieren: $M=3,24 ; S D=0,87)$. Verbesserungsvorschläge der Expert*innen $\mathrm{zu}$ einzelnen Items wurden vor der Haupterhebung berücksichtigt.

Die deskriptiven Daten der Studierendenbefragung zeigen zunächst, dass insgesamt relativ hohe Mittelwerte in den einzelnen Wissensfacetten erreicht wer- den - die durchschnittliche Itemschwierigkeit liegt bei über $50 \%$ (• Tab. 2). Auch zeigt der Range der Mittelwerte, dass keine sehr schweren Items vorliegen. Schließlich kann festgestellt werden, dass die Wissensfacetten, sofern die entsprechenden Items gemeinsam einer Skala zugeordnet werden, relativ zufriedenstellende Reliabilitäten aufweisen. Zwar fällt die interne Konsistenz für die Wissensfacetten Lernende unterstützen $(\alpha=0,60)$ und Schwierigkeiten erklären $(\alpha=0,66)$ nicht besonders hoch aus. Allerdings wurden übliche Faustregeln (etwa $\alpha>0,70$ ) eher für homogenere psychologische Testskalen entwickelt und sollten daher für (heterogenere) Wissenstests moderater interpretiert werden (s. dazu auch Kline, 1999). Die interne Konsistenz der Wissensfacette Vorgehensweisen formulieren ist unauffällig $(\alpha=0,78)$.

\section{Faktorielle Struktur des fachdidaktischen Wissens (Hypothese 1 und 2)}

Zur Überprüfung der faktoriellen Struktur des fachdidaktischen Wissens (Hypothese 1) wird ein 3-Faktor-Modell mit einem G-Faktor-Modell verglichen. Der Vergleich der Modellparameter (- Tab. 3) zeigt, dass das 3-Faktor-Modell sowohl bei den inkrementellen FitIndizes $(\mathrm{CFI}=0,91 ; \mathrm{TLI}=0,91)$ als auch bei den residuenbezogenen Fit-Indizes (RMSEA =0,03; SRMR =0,06) sehr zufriedenstellende bis akzeptable Werte aufweist. Zwar liegen die residuenbezogenen Fit-Indizes auch beim G-FaktorModell in einem noch akzeptablen Bereich $(\mathrm{RMSEA}=0,05 ; \mathrm{SRMR}=0,07)$, dies gilt jedoch nicht für die inkrementellen Fit-Indizes (CFI=0,75; TLI=0,72). Zudem weisen sowohl die signifikant unterschiedlichen $\chi^{2}$-Werte $\left(\Delta \chi^{2}=140,01\right.$; $\Delta d f=3, p<0,001)$ als auch die geringeren deskriptiven Modellparameter des 3-Faktor-Modells (AIC $=-5295,10$;
$\mathrm{BIC}=-5019,86)$ auf die Überlegenheit gegenüber dem G-Faktor-Modell hin (AIC $=-5160,55 ; \mathrm{BIC}=-4896,32)$. Alle Indikatoren im 3-Faktor-Modell weisen signifikante Einzelpfade auf (• Abb. 2).

Hinsichtlich des Zusammenhangs der Faktoren (Hypothese 2) kann zwischen den Faktoren Lernende unterstützen und Schwierigkeiten erklären eine hohe Korrelation festgestellt werden $(r=0,83)$. Die Korrelationen dieser beiden Faktoren mit dem Faktor Vorgehensweisen formulieren fallen geringer aus und zeigen sowohl hinsichtlich des Faktors Lernende unterstützen $(r=0,45)$ als auch hinsichtlich des Faktors Schwierigkeiten erklären $(r=0,48)$ einen mittleren Effekt. Aufgrund der relativ hohen Korrelation der Faktoren Lernende unterstützen und Schwierigkeiten erklären wurde abschließend geprüft, ob ein 2-Faktor-Modell, bei dem die Items dieser beiden Faktoren zusammengefasst werden, dem 3-Faktor-Modell überlegen ist. Dies ist nicht der Fall. Zwar lassen die deskriptiven Modellparameter kein eindeutiges Urteil zu: Beim 2-Faktor-Modell fällt der AIC $(-5288,20)$ zwar etwas höher, der BIC $(-5020,30)$ jedoch marginal kleiner aus als beim 3-Faktor-Modell. Gleichwohl liegt hinsichtlich des $\chi^{2}$-Wertes eine eindeutig (signifikant) bessere Aufklärung der Daten durch das 3-FaktorModell als durch das 2-Faktor-Modell vor $\left(\Delta \chi^{2}=10,36 ; \Delta d f=2, p=0,006\right)$.

\section{Zusammenhänge zur Abiturnote und zum Studiensemester (Hypothese 3)}

Hinsichtlich der Vorhersage durch die Abiturnote bzw. die Semesterzahl (Hypothese 3) kann festgestellt werden: Weder der Faktor Lernende unterstützen $(r=-0,08)$, noch der Faktor Schwierigkeiten erklären $(r=-0,14)$ oder der Faktor Vorgehensweisen formulieren $(r=-0,01)$ wird signifikant durch die Abiturnote vorhergesagt. Hingegen liegen positive 


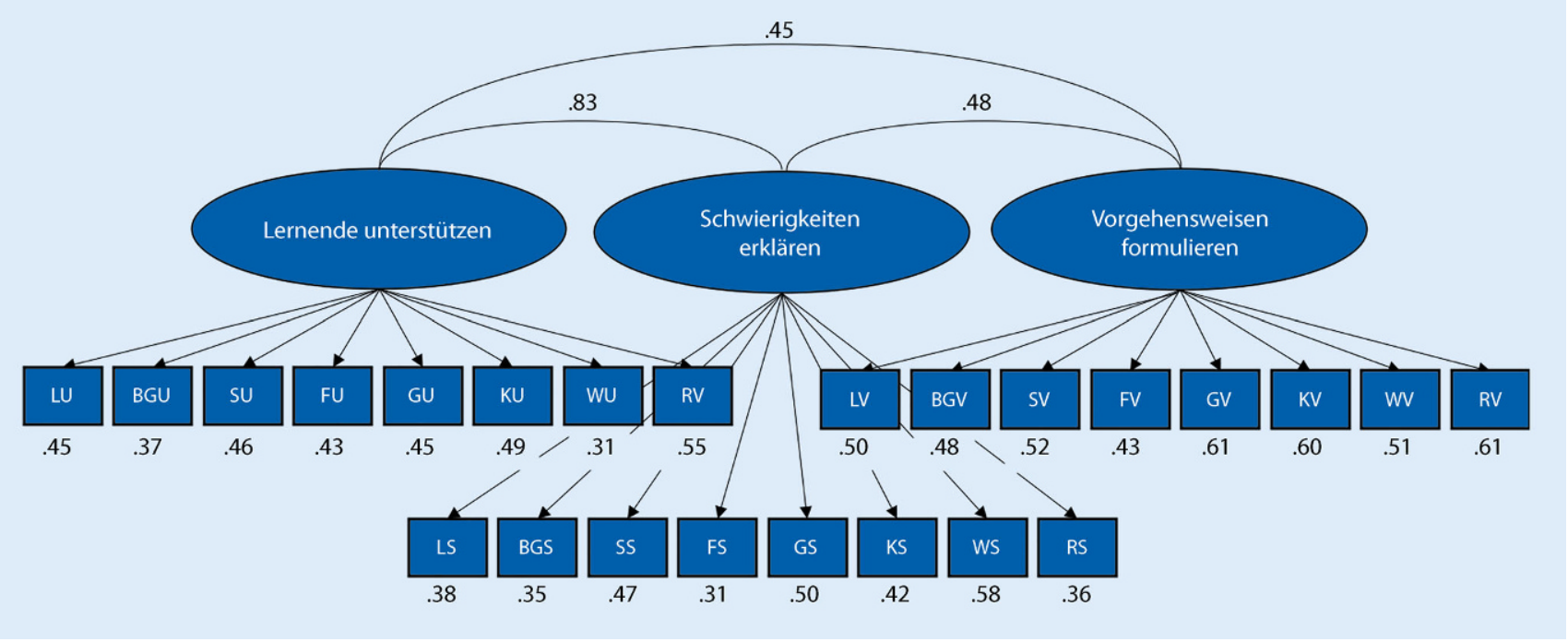

Abb. 2 \& 3-Faktor-Modell zum fachdidaktischen Wissen von angehenden Sportlehrkräften. Anfangsbuchstabe[n]: L Laufen, Springen, Werfen, $B G$ Bewegen an und mit Geräten, $S$ Spielen in und mit Regelstrukturen, $F$ den Körper trainieren, die Fitness verbessern, $G$ Bewegung gestalten, $K$ mit/gegen Partner kämpfen, $W$ Bewegen im Wasser, $R$ Rollen, Gleiten, Fahren; Endbuchstabe: $U$ Lernende unterstützen, $S$ Schwierigkeiten erklären, $V$ Vorgehensweisen erklären

Vorhersagen seitens der Semesterzahl sowohl hinsichtlich des Faktors Lernende unterstützen $\left(r=0,32^{\star *}\right)$ als auch hinsichtlich des Faktors Schwierigkeiten erklären $\left(r=0,26^{\star *}\right)$ und des Faktors Vorgehensweisen formulieren $\left(r=0,26^{*}\right)$ vor.

\section{Diskussion}

\section{Interpretation der Ergebnisse}

Zur Erfassung des fachdidaktischen Wissens von angehenden Sportlehrkräften wurden in der vorliegenden Studie Items entwickelt, die sich zum einen den Wissensfacetten Lernende unterstützen, Schwierigkeiten erklären sowie Vorgehensweisen formulieren und zum anderen acht Bewegungsfeldern für das Fach Sport zuordnen lassen. Es findet somit eine (erweiterte) Orientierung an der fachübergreifend weit verbreiteten Heuristik Shulmans (1986) statt, die bis dato für das Fach Sport in der deutschsprachigen Debatte kaum berücksichtigt wurde (Heemsoth, 2016), gleichwohl sie sich auch mit sportdidaktischen Positionen eines Erziehenden Sportunterrichts (z.B. Prohl, 2017a) gut in Einklang bringen lässt.

Die Expert ${ }^{*}$ innen-Befragung verweist zunächst darauf, dass insgesamt relevan- te Items für den Sportunterricht entwickelt wurden. Die Relevanzeinschätzung liegt in einem Bereich, der vergleichbar etwa mit den Einschätzungen der Expert ${ }^{*}$ innen im FALKO-Projekt ist. Hier liegen die Werte der sechs Fächer (ebenfalls bei einer Skalierung bis 4) im Bereich 3,00-3,68 (Krauss et al., 2017). Auch die Einschätzung der Plausibilität der Items fällt in der vorliegenden Studie zufriedenstellend aus. Insbesondere im Rahmen der geschlossenen Items wird somit dem Umstand Rechnung getragen, dass es „für fachdidaktische Items oft keine eindeutig normativ richtige Antwort gibt“ (Krauss et al., 2017, S. 144); dies scheint insbesondere für das Fach Sport relevant, da sich hier Empfehlungen des Unterrichtens, wie sie auch in bewegungsfeldbezogener Literatur zu finden sind, oftmals auf praktische Erfahrungen - nicht aber stets erhärtete Befunde - beziehen. Es ist dennoch gelungen, Items zu entwickelt, bei denen die Güte von Antworten gut eingeschätzt werden kann.

Hinsichtlich der faktoriellen Struktur kann die Annahme gestützt werden, dass sich das fachdidaktische Wissen von angehendenden Sportlehrkräften durch das theoretisch begründete 3-Faktor-Modell abbilden lässt (Hypothese 1). Während in zahlreichen Ansätzen (anderer
Fächer) zur Erfassung des fachdidaktischen Wissens auf empirischer Ebene Generalfaktormodelle berichtet werden (z.B. Baumert et al., 2010; Lange et al., 2012; Mahler et al., 2017), erweist sich in dieser Studie ein Generalfaktor als nicht adäquat. Alle drei Faktoren hängen bedeutsam miteinander zusammen, worauf die Korrelationen mit mittlerer bis großer Effektstärke hinweisen (Hypothese 2). Inhaltlich könnte eine Erklärung für den stärkeren Zusammenhang zwischen den Faktoren Lernende Unterstützen und Schwierigkeiten erklären sein, dass hier Faktoren abgebildet werden, die eher Aspekte der Lernbegleitung in direkter Lehrer*innenSchüler*innen-Interaktion aufgreifen (s. dazu auch Wibowo, 2016) während der Faktor Vorgehensweisen formulieren eher Aspekte einer umfänglicheren Planung (Scheid \& Prohl, 2017) umfasst (s. dazu auch Blömeke et al., 2010). Vor dem Hintergrund verschiedener Ansätze zur Konstruktion des fachdidaktischen Wissens von Sportlehrkräften liegt auf diese Weise eine der wenigen empirischen Befunde zur faktoriellen Struktur des fachdidaktischen Wissens von angehenden Sportlehrkräften vor.

Hinsichtlich der unterschiedlichen Annahmen zur Entwicklung des fachdidaktischen Wissens stützt die vorliegende 
Studie eher die Qualifikationshypothese (Kunter, Kleickmann, et al., 2013): Das fachdidaktische Wissen der Studierenden wird signifikant (mit kleiner bis mittlerer Effektstärke) nur durch die Semesterzahl vorhergesagt. Dies stützt die Annahme, dass sich fachdidaktisches Wissen erst durch eine elaborierte Auseinandersetzung über Unterrichtspraxis entwickeln kann („Deliberatepratice“-Hypothese, s. Ericsson, 2006), wofür das Studium Lerngelegenheiten anbieten kann. Eine fachspezifische Erklärung könnte zudem sein, dass für den Aufbau fachdidaktischen Wissens auch persönliche Bewegungserfahrungen und -kompetenzen hilfreich sind (s. dazu auch Vogler et al., 2017), die im Rahmen der Abiturnote nur marginal abgebildet werden, sich jedoch im Verlaufe des Studiums verstärkt einstellen (sollten).

\section{Grenzen und zukünftige Forschung}

Der vorliegende Beitrag unterliegt methodischen wie inhaltlichen Grenzen, die als Anlass zukünftiger Forschung gesehen werden können. Zunächst ist aus theoretischer Perspektive festzustellen, dass für die Itementwicklung eine Orientierung an spezifischen Gestaltungsmerkmalen stattfand, deren Reichweite hinterfragt werden kann.

Bezüglich der realistischen Unterrichtsszenarien als zentrales Gestaltungsmerkmal erscheint es zwar plausibel, dass dadurch eine gewisse Nähe zur späteren Unterrichtspraxis hergestellt wird (Baumgartner, 2018a), gleichwohl fehlen empirische Studien, die systematisch unterschiedliche Itemformate vergleichen und auf Stärken und Schwächen unterschiedlicher Ansätze verweisen. Offen ist zudem, inwieweit eine noch stärkere Kontextualisierung von Items zu einer stärkeren prädikativen Validität entsprechender Tests beitragen kann, etwa indem die entsprechenden Unterrichtssituationen in Videoclips abgebildet und unter Zeitdruck bearbeitet werden (etwa Lindmeier, 2011).

Die Fokussierung sportunterrichtlicher Inhalte durch Bewegungsfelder ist eine pragmatische, zugleich aber diskussionswürdige Vorgehensweise, da sie
u.U. den Eindruck erwecken könnte, dass sich Sportunterricht auf motorisch-technische Ziele (innerhalb von Bewegungsfeldern) zu konzentrieren habe. Dabei sei jedoch darauf verwiesen, dass im Rahmen der vorliegenden Testkonstruktion zum fachdidaktischen Wissen von Sportlehrkräften darauf geachtet wurde, dass eine große Breite an sportunterrichtlichen Zielen im Sinne des Doppelauftrags eines Erziehenden Sportunterrichts vertreten ist - gleichwohl wird dieser Aspekt nicht systematisch variiert. Eine Möglichkeit, dies zukünftig zu tun, könnte die Konzentration auf einzelne Bewegungsfelder darstellen. So könnten dann Ziele, etwa für ein bestimmtes Bewegungsfeld, durch pädagogische Perspektiven oder Kompetenzen ausdifferenziert und als Dimensionen der Testkonstruktion verwendet werden.

Schließlich ist $\mathrm{zu}$ betonen, dass die eingenommene Orientierung an der Shulman-Heuristik auf der einen Seite zwar eine Anschlussfähigkeit an andere Fachkonzeptionen ermöglicht, zukünftig jedoch geklärt werden sollte, inwieweit über andersartige Modellierungen (z. B. in Anlehnung an Scherler, 2004; s. Abschn. „Bisherige Konzeptualisierungsansätze für das Fach Sport“) vergleichbare oder auch andere Aspekte fachdidaktischen Wissens von Sportlehrkräften erfasst werden können.

Aus methodischer Perspektive ist festzuhalten, dass sich die relativ hohe Korrelation zwischen den ersten beiden Faktoren in Teilen auch auf einen Effekt des Antwortmodus zurückführen lassen könnte. Denn es sind gerade die Faktoren mit geschlossenen Items, die am höchsten miteinander korrelieren. Inwieweit die Zusammenhänge unabhängig der Art der Items sind, sollte in zukünftiger Forschung geklärt werden. Hier könnte dann auch untersucht werden, inwieweit Veränderungen der Itemschwierigkeit erreicht werden sollten: Die vorliegenden Befunde deuten darauf hin, dass insgesamt ein eher leichter Test vorliegt. Dies kann zum Teil damit erklärt werden, dass Studierende am Ende ihres Bachelorstudiums und Masterstudierende, die aufgrund ihres fortgeschrittenen Ausbildungsstandes auch mehr fach- didaktische Lerngelegenheiten erhalten haben sollten, überdurchschnittlich häufig vertreten waren. Auch für diese Studierendengruppe erscheint es jedoch sinnvoll, schwerere Items zu berücksichtigen, um die Fähigkeiten adäquat abbilden zu können. Darüber hinaus gilt es zukünftig zu prüfen, inwieweit die Itemschwierigkeit ggf. auch von der Bearbeitung als Hausaufgabe beeinflusst ist. Zwar lassen mündliche (nicht jedoch systematisch erhobene) Rückmeldungen der Studierenden hoffen, dass die Aufgaben ohne zusätzlich eingeholte Informationen bearbeitet wurden, ausschließen lässt sich eine entsprechende Verzerrung jedoch nicht.

In diesem Beitrag wurden Strukturgleichungsmodelle für die Analyse der Daten herangezogen, die insbesondere für einen Vergleich verschiedener Modellierungen empfohlen werden. Neben Strukturgleichungsmodellen gelten jedoch auch latenten Modellierungen im Sinne der Item-Response-Theorie (IRT) als zentrale Analyseverfahren bei der Entwicklung von Kompetenztests (Hartig \& Frey, 2013). Insbesondere zur Festlegung von Kompetenzniveaus oder für die Analyse von Daten im MultiMatrix-Design erscheint es zukünftig gewinnbringend, auch dieses Verfahren $\mathrm{zu}$ berücksichtigen.

Das vorgestellte Instrument lädt langfristig schließlich dazu ein, Zusammenhänge zwischen dem fachdidaktischen Wissen und anderen Wissensformen, wie dem Fachwissen, aber auch die prädiktive Kraft des fachdidaktischen Wissens im Hinblick auf das Erreichen von Unterrichtszielen für das Fach Sport zu untersuchen. Dabei erscheint es jedoch plausibel, das fachdidaktische Wissen von Sportlehrkräften noch spezifischer zu betrachten: Werden beispielsweise Lernverläufe von Schüler*innen hinsichtlich ihrer Spielfähigkeit untersucht, so macht es Sinn, (ausschließlich) das fachdidaktische Wissen hinsichtlich der Vermittlung im Bewegungsfeld Spielen in und mit Regelstrukturen zu betrachten und das vorhandene Instrument dafür ggf. zu erweitern (s. dafür etwa auch die engen Konzeptionen im Fach Mathematik; Kunter et al., 2011). 


\section{Korrespondenzadresse}

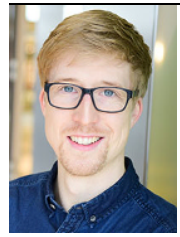

PD Dr. Dr. Tim Heemsoth

Fakultät für Erziehungswissenschaft, Arbeitsbereich Bewegung, Spiel und Sport, Universität Hamburg

Von-Melle-Park 8, 20146 Hamburg, Deutschland tim.heemsoth@ uni-hamburg.de

Funding. Open Access funding provided by Projekt DEAL.

\section{Einhaltung ethischer Richtlinien}

Interessenkonflikt. T. Heemsoth und J. Wibowo geben an, dass kein Interessenkonflikt besteht.

Die Untersuchung wurde im Einklang mit nationalem Recht sowie gemäß der Empfehlungen der DFG zur Sicherung guter wissenschaftlicher Praxis durchgeführt. Für die aufgeführten Studien gelten die jeweils dort angegebenen ethischen Richtlinien.

Open Access Dieser Artikel wird unter der Creative Commons Namensnennung 4.0 International Lizenz veröffentlicht, welche die Nutzung, Vervielfältigung, Bearbeitung, Verbreitung und Wiedergabe in jeglichem Medium und Format erlaubt, sofern Sie den/die ursprünglichen Autor(en) und die Quelle ordnungsgemäß nennen, einen Link zur Creative Commons Lizenz beifügen und angeben, ob Änderungen vorgenommen wurden.

Die in diesem Artikel enthaltenen Bilder und sonstiges Drittmaterial unterliegen ebenfalls der genannten Creative Commons Lizenz, sofern sich aus der Abbildungslegende nichts anderes ergibt. Sofern das betreffende Material nicht unter der genannten Creative Commons Lizenz steht und die betreffende Handlung nicht nach gesetzlichen Vorschriften erlaubt ist, ist für die oben aufgeführten Weiterverwendungen des Materials die Einwilligung des jeweiligen Rechteinhabers einzuholen.

Weitere Details zur Lizenz entnehmen Sie bitte de Lizenzinformation auf http://creativecommons.org/ licenses/by/4.0/deed.de.

\section{Literatur}

Aloe, A. M., \& Becker, B. J. (2009). Teacher verbal ability and school outcomes. Educational Researcher, 38,612-624.

Anderson, L. W., \& Krathwohl, D. R. (2001). A taxonomy for learning, teaching and assessing: a revision of bloom's taxonomy of educational objectives. New York:Longman.

Bandalos, D. L. (2002). The effects of item parceling on goodness-of-fit and parameter estimate bias in structural equation modeling. Structural Equation Modelling, 9, 78-102.

Baumert, J., \& Kunter, M. (2006). Stichwort: Professionelle Kompetenz von Lehrkräften. Zeitschrift für Erziehungswissenschaft, 9,469-520.

Baumert, J., Kunter, M., Blum, W., Brunner, M., Voss, T., Jordan, A., Tsai, Y. M., et al. (2010). Teachers' mathematical knowledge, cognitive activation in the classroom, and student progress. American Educational Research Journal,47, 133-180.

Baumgartner, M. (2018a). .... Kompetenz ohne Performanz ist leer! Performanz ohne Kompetenz blind ...!" Zu einem integrativen Kompetenzstrukturmodell von Sportlehrkräften. Zeitschrift für sportpädagogische Forschung, 6, 49-68.

Baumgartner, M. (2018b). Performanzfortschritt in de Lehrerausbildung durch die Arbeit an eigenen video- und textbasierten Unterrichtsfällen? Eine Interventionsstudie zur Verbesserung des Feedbacks bei angehenden Sportlehrkräften. Zeitschrift für Erziehungswissenschaft, 21, 1135-1155.

Blömeke, S., Gustafsson, J. E., \& Shavelson, R. J. (2015). Beyond dichotomies: competence viewed as a continuum. Zeitschrift für Psychologie, 223, $3-13$.

Blömeke, S., Kaiser, G., \& Lehmann, R. (2010). TEDS-M 2008. Professionelle Kompetenz und Lerngelegenheiten angehender Mathematiklehrkräfte für die Sekundarstufe I im internationalen Vergleich. Münster:Waxmann.

Bromme, R. (2001). Teacher expertise. In N. J. Smelser, P. B. Baltes \& F. E. Weinert (Hrsg.), International encyclopedia of the behavioral sciences: Education (S. 15459-15465). London: Pergamon.

Cohen, J. (1988). Statistical power analysis for the behavioral sciences (2. Aufl.). Hillsdale: Erlbaum

Döring, N., \& Bortz, J. (2016). Forschungsmethoden und Evaluation in den Sozial- und Humanwissen schaften (5. Aufl.). Berlin, Heidelberg: Springer.

Ericsson, K.A. (2006). The influence of experience and deliberate practice on the development of superior expert performance. In K. A. Ericsson, N. Charness, P.J. Feltovich \& R.R. Hoffman (Hrsg.), The Cambridge handbook of expertise and expert performance (S. 685-706). Cambridge: Cambridge University Press.

Förtsch, C., Werner, S., von Kotzebue, L., \& Neuhaus, B. J. (2016). Effects of biology teachers' professional knowledge and cognitive activation on students' achievement. International Journal of Science Education, 38, 2642-2666

Grönqvist, E., \& Vlachos, J. (2008). One size fits all? The effects of teacher cognitive and non-cognitive abilities on student achievement. Uppsala: Institute for Labour Market Policy Evaluation.

Größing, S. (2007). Einführung in die Sportdidaktik (9. Aufl.).Wiebelsheim: Limpert.

Hartig, J., \& Frey, A. (2013). Sind Modelle der ItemResponse-Theorie (IRT) das Mittel der Wahl für die Modellierung von Kompetenzen? Zeitschrift fur Erziehungswissenschaft, 16, 47-51.

Heemsoth, T. (2016). Fachspezifisches Wissen von Sportlehrkräften. Ein Überblick über fachübergreifende und fachfremde Ansätze und Perspektiven für die Professionsforschung von Sportlehrkräften. Zeitschrift für sportpädagogische Forschung, 3, 41-60.

Heemsoth, T., \& Kleickmann, T. (2018). Learning to plan self-controlled physical education: good vs. problematic teaching examples. Teaching and Teacher Education, 71, 168-178.

Heim, R., \& Sohnsmeyer, J. (2015). Schulsport. In W. Schmidt (Hrsg.), Dritter Deutscher Kinder- und Jugendsportbericht (S. 118-139). Schorndorf: Hofmann.

Hill, H. C., Schilling, S. G., \& Ball, D. L. (2004). Developing measures of teachers' mathematics knowledge for teaching. The Elementary School Journal, 105, 11-30.

Kennedy, M. M., Ahn, S., \& Choi, J. (2008). The value added by teacher education. In M. Cochran-
Smith, S. Feiman-Nemser, D.J. McIntyre \& K.E. Demers (Hrsg.), Handbook of research on teacher education (S. 1249-1273). New York: Routledge.

Kirschner, P. A., Sweller, J., \& Clark, R.E. (2006). Why minimal guidance during instruction does not work. Educational Psychologist, 41,75-86.

Kleickmann, T., Großschedl, J., Harms, U., Heinze, A. Herzog, S., Hohenstein, F., Zimmermann, F., et al. (2014). Professionswissen angehender Lehrkräfte mit mathematisch-naturwissenschaftlichen Fächern - Testentwicklung im Rahmen des Projekts KiL. Unterrichtswissenschaft, 42, 280-288.

Kleickmann, T., Richter, D., Kunter, M., Elsner, J., Besser M., Krauss, S., \& Baumert, J. (2013). Teachers' content knowledge and pedagogical content knowledge: the role of structural differences in teacher education. Journal of Teacher Education, $64,90-106$.

Kline, P. (1999). The handbook of psychological testing (2. Aufl.).London: Routledge.

Krauss, S. (2011). Das Experten-Paradigma in der Forschung zum Lehrerberuf. In E. Terhart, H. Bennewitz \& M. Rothland (Hrsg.), Handbuch der Forschung zum Lehrerberuf (S. 171-191). Münster:Waxmann

Krauss, S., Lindl, A., Schilcher, A., Fricke, M., Göhring, A., Hofmann, B., Mulder, H., et al. (2017). FALKO. Fachspezifische Lehrerkompetenzen. Münster: Waxmann.

Kuckartz, U. (2016). Qualitative Inhaltsanalyse. Methoden, Praxis, Computerunterstützung (3. Aufl.). Weinheim: Beltz.

Kunter, M., Baumert, J., Blum, W., Klusmann, U., Krauss, S., \& Neubrand, M. (2011). Professionelle Kompetenz von Lehrkräften: Ergebnisse des Forschungsprogramms COACTIV. Münster: Waxmann.

Kunter, M., Kleickmann, T., Klusmann, U., \& Richter, D. (2013a). The development of teachers' professional competence. In M. Kunter, J. Baumert, W. Blum, U. Klusmann, S. Krauss \& M. Neubrand (Hrsg.), Cognitive activation in the mathematics classroom and professional competence of teachers. Results from the COACTIV project(S.63-77) New York: Springer.

Kunter, M., Klusmann, U., Baumert, J., Richter, D., Voss, T., \& Hachfeld, A. (2013b). Professional competence of teachers: effects on instructional quality and student development. Journal of Educational Psychology, 105, 805-820.

Laging, R. (2009). Unterrichten in Bewegungsfeldern. Eine Einführung. In R. Laging (Hrsg.), Inhalte und Themen des Bewegungs- und Sportunterrichts (S. 89-95). Baltmannsweiler: Schneider Hohengehren.

Lange, K., Kleickmann, T., Tröbst, S., \& Möller, K. (2012) Fachdidaktisches Wissen von Lehrkräften und multiple Ziele im naturwissenschaftlichen Sachunterricht.Zeitschrift für Erziehungswissenschaft 15, 55-75.

Lindmeier, A.M. (2011). Modeling and measuring knowledge and competencies of teachers. Münster:Waxmann.

Little, T. D., Cunningham, W. A., Shahar, G., \& Widaman, K. F. (2002). To parcel or not to parcel. Exploring the question, weighing the merits. Structural Equation Modeling, 9, 151-173.

Mahler, D., Großschedl, J., \& Harms, U. (2017). Using doubly latent multilevel analysis to elucidate relationships between science teachers' professional knowledge and students' performance. International Journal of Science Education, 39, 213-237. 
Meier, S. (2018). Fachdidaktisches Wissen angehender Sportlehrkräfte. Ein Konzeptualisierungsvorschlag. Zeitschrift für sportpädagogische Forschung, 6, 69-84.

Muthén, B. O., \& Muthén, L. K. (2012). Mplus. User manual. Los Angeles: Muthén \& Muthén.

Ohle, A., Fischer, H.E., \& Kauertz, A. (2011). Der Einfluss des physikalischen Fachwissens von Primarstufenlehrkräften auf Unterrichtsgestaltung und Schülerleistung. Zeitschrift für Didaktik der Naturwissenschaften, 17, 357-389.

Prohl, R. (2017a). Der Doppelauftrag des Erziehenden Sportunterrichts. In V. Scheid \& R. Prohl (Hrsg.), Sportdidaktik (2. Aufl. S. 64-84). Wiebelsheim: Limpert.

Prohl, R. (2017b). Vermittlungsformen im Erziehenden Sportunterricht. In V. Scheid \& R. Prohl (Hrsg.), Sportdidaktik (2. Aufl. S. 85-103). Wiebelsheim: Limpert.

Prohl, R. \& Krick, F. (2006). Lehrplan und Lehrplanentwicklung - Programmatische Grundlagen des Schulsports. In DSB (Hrsg.), DSB-SPRINT-Studie. Eine Untersuchung zur Situation des Schulsports in Deutschland (S. 19-52). Aachen: Meyer \& Meyer.

Questback Gmb, H. (2018). EFS Survey, Version Spring 2018. Köln: Questback GmbH.

Scheid, V., \& Prohl, R. (2017). Sportdidaktik (2. Aufl.) Wiebelsheim: Limpert.

Scherler, K. (2004). Sportunterricht auswerten. Eine Unterrichtslehre. Hamburg: Czwalina.

Schermelleh-Engel, K., Moosbrugger, H., \& Müller, H. (2003). Evaluating the fit of structural equation models: tests of significance and descriptive goodness-of-fit measures. Methods of Psychological Research Online, 8, 23-74.

Shulman, L. S. (1986). Those who understand: knowledge growth in teaching. Educational Researcher, 15, 4-14.

Shulman, L.S. (1987). Knowledge and teaching. Foundations of the new reform. Harvard Educational Review, 57, 1-22.

Vogler, J., Messmer, R., \& Allemann, D. (2017). Das fachdidaktische Wissen und Können von Sportlehrpersonen (PCK-Sport). German Journal of Exercise and Sport Research, 47, 335-347.

Wibowo, J. (2016). Adaptive Lernbegleitung im Sportunterricht. Zeitschrift für sportpädagogische Forschung, 1,63-84.

Wibowo, J., \& Heemsoth, T. (2019). Fachdidaktisches Wissen von Sportlehrer*innen testen: Überlegungen zur Inhaltsvalidität. Zeitschrift für sportpädagogische Forschung, 7, 88-108.

Yeh, S.S. (2009). The cost-effectiveness of raising teacher quality. Educational Research Review, 4, 220-232. 